\title{
SYMPATHETIC COOLING OF LITHIUM BY LASER-COOLED CESIUM
}

\author{
S. KRAFT, M. MUDRICH, K. SINGER, R. GRIMM, A. MOSK AND \\ M. WEIDEMÜLLER \\ Max-Planck-Institut für Kernphysik, 69117 Heidelberg, Germany \\ E-mail: m.weidemueller@mpi-hd.mpg.de
}

\begin{abstract}
We present first indications of sympathetic cooling between two neutral trapped atomic species. Lithium and cesium atoms are simultaneously stored in an optical dipole trap formed by the focus of a $\mathrm{CO}_{2}$ laser, and allowed to interact for a given period of time. The temperature of the lithium gas is found to decrease when in thermal contact with cold cesium. The timescale of thermalization yields an estimate for the Li-Cs cross-section.
\end{abstract}

Mixtures of atomic gases enrich the field of physics with ultracold gases by making many interesting phenomena available for experimental study. A prime example is the exchange of thermal energy between different components in the mixture. This cross-thermalization can be used for determining inter-species collision cross-sections, and, more importantly, for sympathetic cooling. The process of sympathetic cooling, where the gas of interest is cooled through thermalization with a coolant gas, is especially advantageous if the cooled gas ( $\mathrm{Li}$ in our case) is hard to cool by other methods, while the coolant gas (Cs) allows for efficient laser cooling. The Li can only be optically cooled to approximately the Doppler temperature of $0.14 \mathrm{mK}$, and evaporative cooling of this atom in its stable $F=1$ ground state is extremely difficult due to its anomalously low scattering length 1 . Cesium, on the other hand, allows sub-Doppler temperatures $(<3 \mu \mathrm{K}$ in free space) to be reached by simple polarization-gradient cooling. This opens the perspective of reaching a stable Bose-Einstein condensate (BEC) of $\mathrm{Li}$ in the $F=1$ state (as in the experiment by Schreck et al. 4 ) without evaporative cooling.

Our apparatus is described in detail in a previous paper 6 . The thermalization experiments take place in an optical dipole trap, which is based on a $\mathrm{CO}_{2}$ laser emitting light at $10.6 \mu \mathrm{m}$. As the laser frequency is detuned far below any optical resonances of the atoms, this trap is referred to as a quasielectrostatic trap (QUEST) 3. Our QUEST employs a $140 \mathrm{~W}$ laser beam which is focused to a beam waist of $90 \mu \mathrm{m}$. This yields a depth of $0.4 \mathrm{mK}$ for $\mathrm{Li}$ and $1.0 \mathrm{mK}$ for $\mathrm{Cs}$. The axial and radial oscillation frequencies for Cs

*PERMANENT ADDRESS: UNIVERSITY OF INNSBRUCK, A-6020 INNSBRUCK, AUSTRIA

icols: submitted to World Scientific on October 28, 2018 


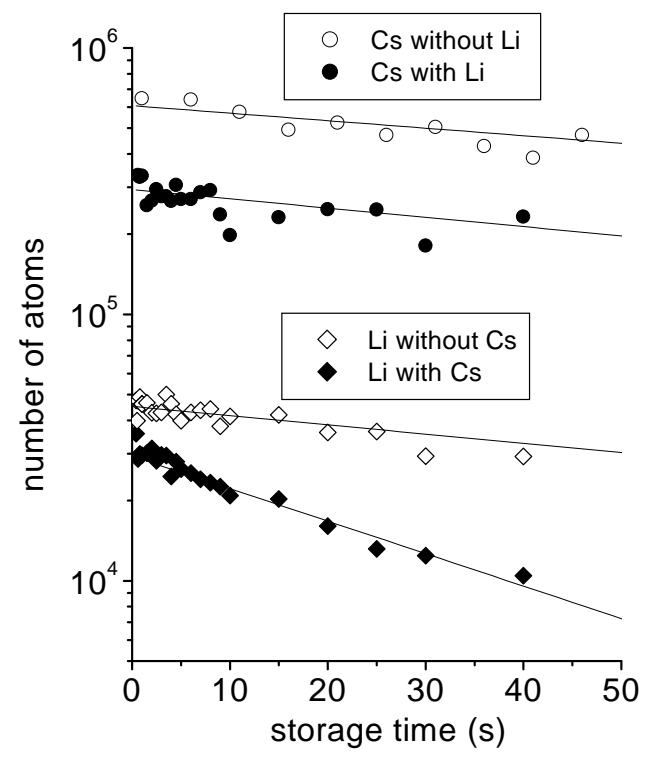

Figure 1. Evolution of the number of trapped $\mathrm{Li}(\mathrm{F}=1)$ and $\mathrm{Cs}(\mathrm{F}=3)$ atoms in simultaneous trapping experiments (closed symbols). For comparison, the open symbols show the evolution of separately trapped gases. Thin lines: Exponential fits. Time constants are 150 s for Cs with or without Li, $125 \mathrm{~s}$ for Li without Cs, and $35 \mathrm{~s}$ for Li trapped simultaneously with Cs.

atoms are 18 and $800 \mathrm{~Hz}$, respectively, the corresponding frequencies for $\mathrm{Li}$ are higher by a factor 2.8 due to the difference in mass and polarizability.

The atoms are loaded into the QUEST from a combined magneto-optical trap (MOT) 1 . We load up to $10^{6} \mathrm{Cs}$ atoms, at a temperature of $\sim 30 \mu \mathrm{K}$ and up to $10^{5} \mathrm{Li}$ atoms, which have a thermal energy of the order of the trap depth.

In a typical experiment, we trap a mixed sample and let the atoms interact for a variable time, and then turn off the QUEST. We subsequently measure the temperature and density of the Cs gas by absorption imaging (projection of the shadow of the gas cloud on a camera). The Li particle number is measured by fluorescence measurements after recapture in the MOT. The optical density of the Li gas is insufficient to use absorption imaging, therefore the temperature of the Li cannot be determined directly. The evolution of the number of atoms in the trap is shown in Fig. 1. When stored separately, both $\mathrm{Li}$ and $\mathrm{Cs}$ gases decay due to rest gas collisions in 125 and $150 \mathrm{~s}$, re- 


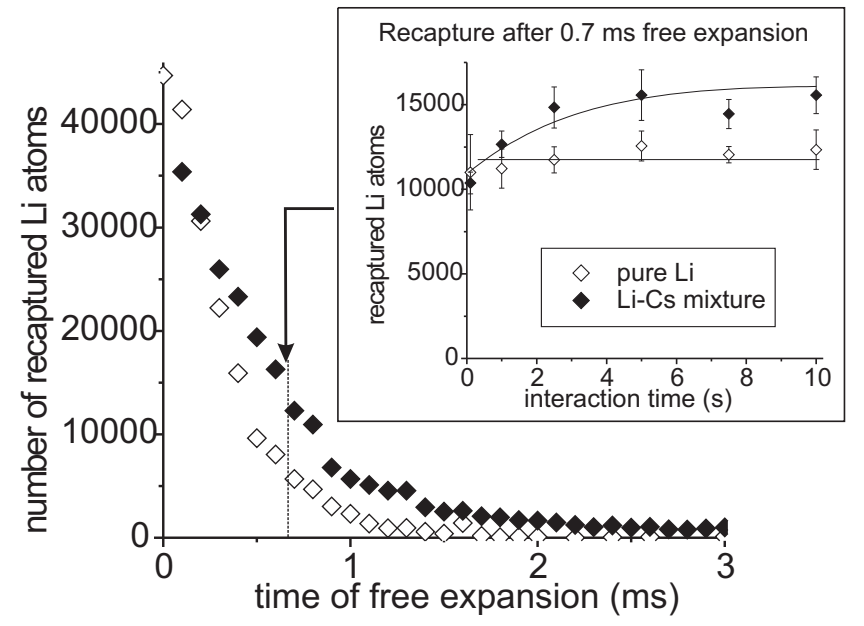

Figure 2. Main graph: Example of release-recapture measurements. The number of recaptured lithium atoms is plotted against the free expansion time. Open symbols: a Li sample just after loading from the MOT. Closed symbols: a sympathetically cooled Li sample (interaction time $15 \mathrm{~s}$ ). Inset: Evolution of the number of recaptured atoms (free expansion time $0.7 \mathrm{~ms})$. Closed symbols: number of recaptured Li atoms after sympathetic cooling. Open symbols: reference measurement with no Cs present. The lines serve as a guide to the eye.

spectively. There is no indication of evaporation in either case: Cs does not evaporate as its temperature is far below the trap depth. Li evaporation is energetically possible but the cross section for $\mathrm{Li}-\mathrm{Li}$ collisions is so small that the characteristic timescale is $>1000 \mathrm{~s}$.

When the gases are stored simultaneously, the Li atom number shows a non-exponential initial behavior followed by an approximately exponential decay with a characteristic time of $35 \mathrm{~s}$, which is much faster than the $125 \mathrm{~s}$ rest gas induced decay. We interpret this as evaporation due to elastic collisions with the Cs. From the evolution of the particle number we can determine inelastic and elastic heteronuclear collision rates 2 . However, the clearest signature of sympathetic cooling, reduction of the temperature of the cooled gas, cannot be obtained this way.

To determine the lithium temperature change due to sympathetic cooling, we have performed release-and-recapture measurements, in a manner analogous to experiments by O'Hara et al. 5. In this type of measurement, the QUEST is turned off and the atoms are released in free space for a short time. 
After a free expansion time of up to $2 \mathrm{~ms}$ the QUEST is turned on again. The fraction of $\mathrm{Li}$ atoms that were recaptured in the QUEST is a measure of the $\mathrm{Li}$ temperature, since slow atoms take a longer time to leave the trapping region. A more quantitative analysis of these release-recapture measurements is being performed and will be published elsewhere.

In figure 2 release-recapture thermometry is demonstrated on two different Li samples: one which has been loaded from the MOT and stored in the QUEST for a short time without Cs, and one which has been stored together with a cold Cs sample for $15 \mathrm{~s}$. The figure clearly shows that in the latter case more atoms are recaptured at long times, i.e., a larger number of slow atoms is present. This indicates that the lithium is cooled sympathetically by the laser cooled cesium atoms.

The timescale of thermalization can be estimated from the inset in Fig. 2, where a single point from the release-recapture curve was measured for different interaction times. A thermalization timescale of roughly 3 seconds can be estimated. From a rate equation model 1 one estimates elastic scattering cross-section of $\sigma_{\mathrm{CsLi}} \sim 3 \times 10^{-12} \mathrm{~cm}^{2}$ which is in satisfactory agreement with the earlier estimate we obtained from evaporation measurements 6 . Note that the absolute number of slow atoms increases during thermalization, indicating an increasing phase space density.

Further efforts will concentrate on lowering the temperature of the coolant gas and increasing the number of trapped $\mathrm{Li}$ atoms, thereby increasing the degeneracy parameter. By repeated pulsed optical cooling one can extract the entropy that the Li transfers to the Cs, thereby providing a truly lossless cooling mechanism towards BEC of Li. For a sample of $10^{6} \mathrm{Li}$ atoms, the BEC transition temperature is $\sim 3 \mu \mathrm{K}$ in our trap.

This work has been supported in part by the Deutsche Forschungsgemeinschaft. A.M. is supported by a Marie-Curie fellowship from the European Community programme IHP under contract number CT-1999-00316. We are indebted to D. Schwalm for encouragement and support.

\section{References}

1. F. Schreck et al., Phys. Rev. Lett. 87, 080403 (2001).

2. A. Mosk et al., submitted to Appl. Phys. B; arXiv:physics/0107075

3. T. Takekoshi and R. J. Knize, Opt. Lett. 21, 77 (1996).

4. U. Schlöder et al., Eur. Phys. J. D 7, 331 (1999).

5. K. M. O'Hara et al., Phys. Rev. Lett. 85, 2092 (2000). 